\title{
A Method to Estimate the Horizontal Handover Decision Effect on Indoor Wireless Conversational Video Quality
}

\author{
Alfonso Fernandez Duran, ${ }^{1}$ Raquel Perez Leal, ${ }^{2}$ and Jose I. Alonso ${ }^{2}$ \\ ${ }^{1}$ Alcatel-Lucent Spain, Ramirez de Prado 5, 28045 Madrid, Spain \\ ${ }^{2}$ Escuela Tecnica Superior de Ingenieros de Telecomunicacion, Universidad Politecnica de Madrid, Ciudad Universitaria, \\ 28040 Madrid, Spain \\ Correspondence should be addressed to Alfonso Fernandez Duran, alfonso.fernandez_duran@alcatel-lucent.es \\ Received 1 October 2007; Revised 31 January 2008; Accepted 4 April 2008 \\ Recommended by Jianfei Cai
}

\begin{abstract}
One of the most interesting and valuable services considered in fixed mobile convergence is video telephony. The success of this conversational video service will depend on the conversational video quality achieved in the multicell wireless indoor scenarios. One of the essential elements in the quality is the effect of the horizontal handovers in the conversational video. This paper analyzes the handover decision based on the probability calculation of handover events in the case of relative signal strength with hysteresis threshold (RSSHT) approach, and it proposes a new handover decision mechanism, variable hysteresis, to avoid unnecessary handovers. The paper presents the impact of the number of handovers and their duration time on the video's effective frame rate. Moreover, the effect of video stream modification during a short handover is also analyzed. Probability and handover duration approaches are combined and a new simple method for video quality evaluation is caused by the handovers in multicell indoor WLAN scenarios. Finally, the model proposed has been applied to a real office scenario.
\end{abstract}

Copyright ( 12008 Alfonso Fernandez Duran et al. This is an open access article distributed under the Creative Commons Attribution License, which permits unrestricted use, distribution, and reproduction in any medium, provided the original work is properly cited.

\section{INTRODUCTION}

In the current context of fixed-mobile convergence, WLAN technology based on 802.11 is becoming available in common portable and mobile user terminals. This has brought about the possibility of using WLAN technology in conversational applications. Although IEEE802.11 was originally intended to transport best-effort data traffic, the incorporation of new standards like IEEE802.11e has brought about the opportunity of deploying delay and bandwidth sensitive services, like real-time voice and video communications. In these circumstances, WLANs combined with IP are being used as technology for limited mobility and nomadic services. The success of this scenario will depend on maintaining the communication's continuity through networks with several wireless access points (APs) by means of horizontal handover. WLANs were not initially designed to support handover between access points, based on the fact that users will most probably remain within the networks in a rather stationary way, using nonreal-time services. The normal use of a WLAN typically supports nonreal-time handovers between APs, provided that the users have access rights to the destination network. The handover consists of an association to a wireless AP once the network client enters the coverage area of the destination AP. The horizontal handover is therefore a break before make process. User terminals usually incorporate just one WLAN transceiver, but in the case where two transceivers could be used, [1] describes a mechanism to manage handovers based on the voice over IP packet transmission retries. Horizontal handover is addressed in IEEE802.11r, [2]. The first dealing with authorization issues between different networks, and the second with increasing speed in the handover between access points.

The horizontal handover process could be split into two steps. The first one is to decide whether a handover is necessary and then select the destination AP. The second covers the layer 2 and layer 3 processes. The first step could take place in parallel with the communication without affecting it, while the second takes time from the service being conveyed. A description of the message transactions and time requirements is described in detail in [3]. According 
to [1] layer 2 could take between 50 milliseconds and 400 milliseconds, while layer 3, depending on the network settings could take 300 milliseconds or more.

Several studies have analyzed the handover decision process in cellular communications. Studies of the propagation parameters and criteria followed in handover decisions in cellular networks could be found in [4-7]. These studies are mainly related to outdoor mobile communications. Other studies characterize the performance of the handover in WLANs as based on measurements like [8], that characterize the timings and data transfers between network elements, and [9] measure the effects of handovers in voice communications. The impact of the horizontal and vertical handovers in voice communications is studied in [10] using the E model from ITU G.107.

Regarding video services, as a consequence of the evolution of the technologies and applications, advanced coding techniques have been introduced as a video coding format. In this study, ITU-T Rec. H.264|ISO/IEC 1449610 and H.264 have been considered. H.264 "represents an evolution of the existing video coding standards (H.261, H.262, and H.263) and it was developed in response to the growing need for higher compression of moving pictures for various applications such as videoconferencing, digital storage media, television broadcasting, Internet streaming, and communication" [11].

The H.264 defines a limited subset of syntax called "profiles" and "levels" in order to facilitate video data interchange between different applications. A "profile" specifies a set of coding tools or algorithms that can be used in generating a conforming bitstream, whereas a "level" imposes constraints on certain key parameters of the bitstream. The recommendation defines seven profiles (Baseline, Extended, Main and four High-profile types) and fifteen "levels" per "profile." The same set of "levels" is defined for all "profiles."

Current studies show that handovers have an impact on the quality of communications, since handovers produce discontinuities in the communication data streams. A discussion on video, the packet sizes and the implications in the PSNR are presented in [12]. The results shown are based only on simulations, and no model is proposed to predict the system performance. A qualitative study of using an intelligent access point handover mechanism in WLAN to obtain user perception for video conferencing quality, before and after applying the intelligent access point handover mechanism is presented in [13]. This reference shows that some strategies could improve the handover performance when the APs are congested, however no models are proposed to predict the effect on the video communications performances. In the case of conversational video, it is possible to obtain a simple video quality estimator using the effective frame rate resulting from the packet losses due to the handover effect, as introduced in reference [14].

Therefore, it is very appropriate to analyze the impact of handover on video communications quality in order to better understand the process involved. Moreover, it is also necessary to improve the method of planning real handover scenarios while maintaining the communication quality. The conversational video degradation due to the handover processes taking place in a wireless network can be addressed from at least two general perspectives: video processing to minimize whatever effect is taking place in the transmission media, and wireless processing carried out in the wireless part. With regard to the radio part, a new decision handover mechanism has been proposed (called variable hysteresis) to reduce unnecessary handovers. Moreover, in the video part, video stream modification has been introduced to minimize the handover duration impact arising from the interdependency of video frames. The following sections introduce the framework for conversational video application handover, a new and simple method of conversational video quality estimation based on handover time duration is also proposed. Finally, quality evaluation is shown in a real office scenario and planning recommendation provided.

\section{WLAN HANDOVER PRINCIPLES}

WLANs belonging to the IEEE802.11 family were not originally conceived to support a fast handover between access points. This has become a drawback when deploying multi-AP networks that convey real-time conversational services like IP-based video and voice telephony. The issue comes from the fact that the time necessary to associate it to a new AP is neither controlled nor limited to shorttime intervals. The association time could be of several hundreds of milliseconds as shown in $[8,15]$, while quality of communications such as voice could be severely affected by handover times of more than 50 milliseconds [10]. In addition, the availability of resources at the destination AP is not known until the handover has taken place. To solve these issues, the IEEE802.11r workgroup was set up to define a protocol to enable a fast and reliable handover between access points. By means of this new protocol, the mobile node (MN) can establish security and QoS status before taking a transition decision.

IEEE $802.11 \mathrm{u}$ is another IEEE task group that was set up to allow devices to interconnect with external networks, as typically found in hotspots. The main goal of this task group is to produce an amendment to the IEEE 802.11 standard to allow a common approach to interconnecting IEEE 802.11 access networks to external networks in a generic and standardized manner. The main particular issues covered are network selection, emergency call support, authorization from subscriber network, and media independent handover support.

\subsection{Signal strength}

To analyze the handover process in WLANs, it is necessary to understand the behavior of the received signal strength. In complex propagation scenarios, such as indoors, small changes in spatial separation between wireless access points and observation points impact causing dramatic changes in the signal amplitude and phase. In typical cellular communications systems, the signal strength analysis is based on the long distance outdoor or combined scenarios that experience Rayleigh fading. Several handover studies assume that the fading can be averaged to make up a random 
variable following a lognormal distribution as described in, $[4,5,16,17]$ in the following form:

$$
f_{i}(\widehat{s})=\frac{1}{\hat{s} \sigma_{i} \sqrt{2 \pi}} e^{-\left(\hat{s}-\mu_{i}^{\prime}\right)^{2} / 2 \sigma_{i}^{2}},
$$

where $\hat{s}$ is the received signal amplitude of the envelope, $\mu_{i}^{\prime}$ are the average signal losses received at the mobile node from the wireless access point $i$, and could be expressed as

$$
\mu_{i}^{\prime}=k_{1}+k_{2} \log \left(d_{i}\right)
$$

where $d_{i}$ represents the distances from the observation point to the wireless access point $i, \mathrm{AP}_{i}$. Constants $k_{1}$ and $k_{2}$ represent frequency dependent and fixed attenuation factors, and the propagation constant, respectively. Finally, $\sigma_{i}$ represents the shadowing that could be reasonably averaged to express slow power variations.

Although (1) represents the fading probability distribution function for the path losses as described in (2), in complex scenarios, such as indoors, in which many obstacles make up the propagation losses, the signal strength could be expressed as

$$
\mu_{i}=P_{\mathrm{tx}}-\left(k_{1}+\sum_{k} \lambda_{k}+k_{2} \log \left(d_{i}\right)\right)
$$

where $\lambda_{k}$ is the attenuation of the $k$ passing through walls in the path from the observation point to the wireless access point, and $P_{\mathrm{tx}}$ the transmitted power. Moreover, to take the attenuation into account due to different floors in indoor propagation, one additional term could be added to (3) as stated in [18].

Other possible choices of statistical distributions for modeling the envelope have been described and detailed studies, based on exhaustive measurements, have been carried out to characterize indoor propagation. The Weibull distribution appears to be one of the statistical models that best describes the fading amplitude and fading power indoor scenarios, [18-21] , improving the lognormal distribution in many cases. The Weibull distribution could be expressed as

$$
f_{i}(\hat{s})=b a^{-b} \hat{s}^{b-1} e^{-(\hat{s} / a) b} I_{(0, \infty)}(\hat{s}),
$$

where $\hat{s}$ represents the fading amplitude envelope or the fading power, and $a$ and $b$ are the position and shape values of the distribution, respectively. When the power distribution is represented in $\mathrm{dBm}$, the extreme value distribution function should be used. In fact, if $\hat{s}$ has a Weibull distribution with parameters $a$ and $b, \log (\hat{s})$ has an extreme value distribution with parameters $\mu=\log (a)$ and $\sigma=1 / b$ as shown in $[22,23]$.

The extreme value function for the power probability distribution function (pdf) has been seen as a good approximation. An example of fitting is shown in Figure 1. As can be seen, the power histogram of an indoor trajectory, modeled by the lognormal pdf function, is sufficiently represented by the extreme value function.

This behavior has already been observed in scenarios with complex propagation conditions such as vegetation obstacles [24].

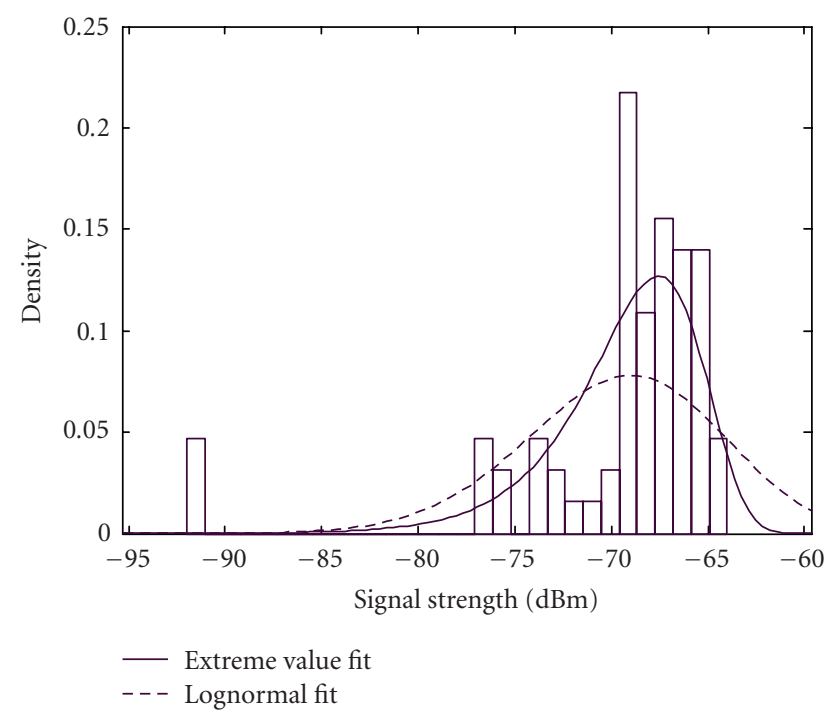

Figure 1: Comparison of lognormal and extreme value pdf fit for an indoor trajectory power log.

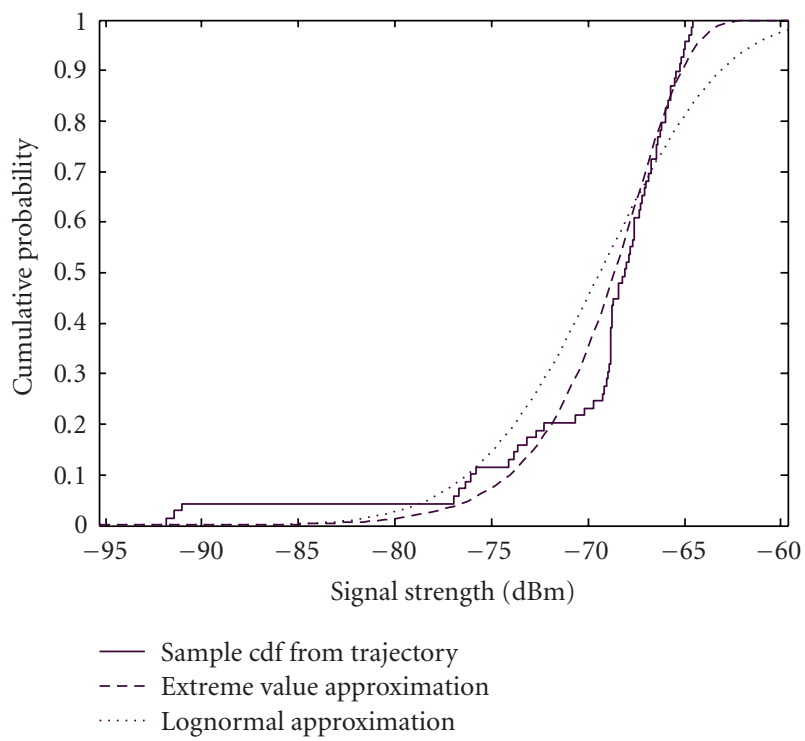

Figure 2: Comparison of lognormal and extreme-value cumulative distribution approximation for an indoor trajectory power log.

Since most of the analysis will be probabilistic, it is interesting to see how the histogram in Figure 1 is approximated in terms of cumulative distribution function (cdf). The comparison results are shown in Figure 2. Integrating the differences between the sample data and the cdf approximations, an overall error of $2 \%$ can be seen for the case of lognormal function, and 5.5\% for the case of extreme value function. Although lognormal is better overall in this scenario, local analysis shows that the maximum difference between sample data and lognormal cdf is 0.24 , while the maximum difference for the extreme value is 0.16 . This allows us to consider extreme value as a reasonable approximation. The use of this approximation will allow 
us to derive analytical expressions for the handover factors involved.

The extreme value probability distribution function is commonly used in the modeling and analysis of phenomena with low occurrence probabilities, as in risk analysis or the study of meteorology.

The pdf of the extreme value distribution can be expressed as

$$
f_{i}(\hat{s})=\frac{1}{\sigma_{i}} e^{\left(\hat{s}-\mu_{i}\right) / \sigma_{i}} e^{-e^{\left(\hat{s}-\mu_{i}\right) / \sigma_{i}}}
$$

where $\sigma_{i}$ and $\mu_{i}$ are as defined above.

To analyze the strategies for the handover decision in outdoor cellular communications in the case of microcells and macrocells, $[4,6,16]$ base their analysis on the estimation of the probability of unnecessary handovers from the statistical power distributions. For example, in the case of two base stations, what would be the probability of handover from base station 1 to 2 and then from 2 back to 1 . In the case of indoor WLAN deployments, the conditions of simultaneous coverage of several access points, in which unnecessary handovers could take place between several of them, for instance from AP1 to AP2, from AP2 to AP3, and the back to AP1 are very frequent. The estimation of handover efficiency based on unnecessary handovers then becomes very complicated, as unnecessary handovers are very difficult to distinguish from necessary ones using the transition logic.

An alternative way of analyzing the handovers in outdoor communications is by means of the residence time, that is, once a handover has taken place, how long the mobile node stays in the new base station. In [25], the residence time statistics for handovers are analyzed. In the case of an indoor WLAN, the size of the cells also makes it very difficult to distinguish handovers based on the residence time, since a normal walking speed could produce a relatively high number of valid handovers with a relatively low residence time.

\subsection{Handover decision techniques}

As an alternative to the analysis carried out for cellular communications, the present study proposes a simple analysis of the handover probability founded on the metrics used to make the handover decision in indoor WLAN communications. It is assumed that the scenario has been properly planned to provide sufficient coverage (minimum signal strength guaranteed at least in one of the access points). The strategy providing a lower probability of handover will present an overall better performance from the communication quality point of view. Based on these principles, several techniques could be used to implement the handover decision. In the following sections, these techniques are presented in order to introduce progressively the mathematical expressions to be used later in the method proposed.

\subsubsection{Best server handover}

If a mobile node sees the power levels $s_{i}$ from each access points $\mathrm{AP}_{i}$ within the coverage range, if no other criteria are implemented, it will associate itself to the one with higher average power. Assuming that the mobile node is currently at $\mathrm{AP}_{0}$, it will hand over to another $\mathrm{AP}_{i}$ if there is one such as $s_{i}>s_{0}$. In this case, the mobile node will carry out handovers on best server basis.

The probability of a handover taking place will be

$$
P_{\mathrm{HO}}=\sum_{i=1}^{n} P_{i}\left(s_{i}>s_{0}\right) .
$$

Since the cumulative distribution function of (5) has a closed form

$$
F(\hat{s})=1-e^{-e^{(\hat{s}-\mu) / \sigma}}
$$

Therefore,

$$
\operatorname{Prob}\left(\hat{s}>s_{0}\right)=1-\operatorname{Prob}\left(s<s_{0}\right) .
$$

Consequently,

$$
\operatorname{Prob}\left(\widehat{s}>s_{0}\right)=1-\left(1-e^{-e^{\left(s_{0}-\mu\right) / \sigma}}\right)=e^{-e^{\left(s_{0}-\mu\right) / \sigma}} .
$$

Combining (6) and (9), the probability of having a handover will be given by

$$
P_{\mathrm{HO}}=\sum_{i=1}^{n} e^{-e^{\left(s_{0}-\mu_{i}\right) / \sigma_{i}}},
$$

where the mobile node is associated to $\mathrm{AP}_{0}$ and simultaneously receives a signal strength above the sensitivity threshold from $n$ access points $\left\{\mathrm{AP}_{1}, \mathrm{AP}_{2}, \ldots \mathrm{AP}_{n}\right\}$.

The main advantage of the best server approach is that it always keeps the mobile node associated to the AP giving the best signal quality, and therefore the higher bandwidth. On the other hand, the main disadvantage is that the number of handovers taking place during a mobile node moving trajectory could be very high, and therefore communications quality issues could take place, especially, in real-time conversational services.

Practical handover decision approaches usually require a reduction in the number of handovers taking place, while keeping the signal strength as high as possible.

\subsubsection{Handover with fixed Hysteresis}

A common approach to reduce the total number of handovers is to use a fixed hysteresis. The mechanism consists of carrying out a handover to a new AP, when the signal strength has improved over certain $h$ value. If the current $\mathrm{AP}$ has a signal strength $s_{0}$, the handover to the new $\mathrm{AP}_{i}$ will happen if $s_{i}>s_{0}+h$.

Figure 3 shows the basic behavior of the hysteresis handover. While the best server approach will produce a handover decision at point $A$, hysteresis approach will produce the decision to handover to the destination AP at 


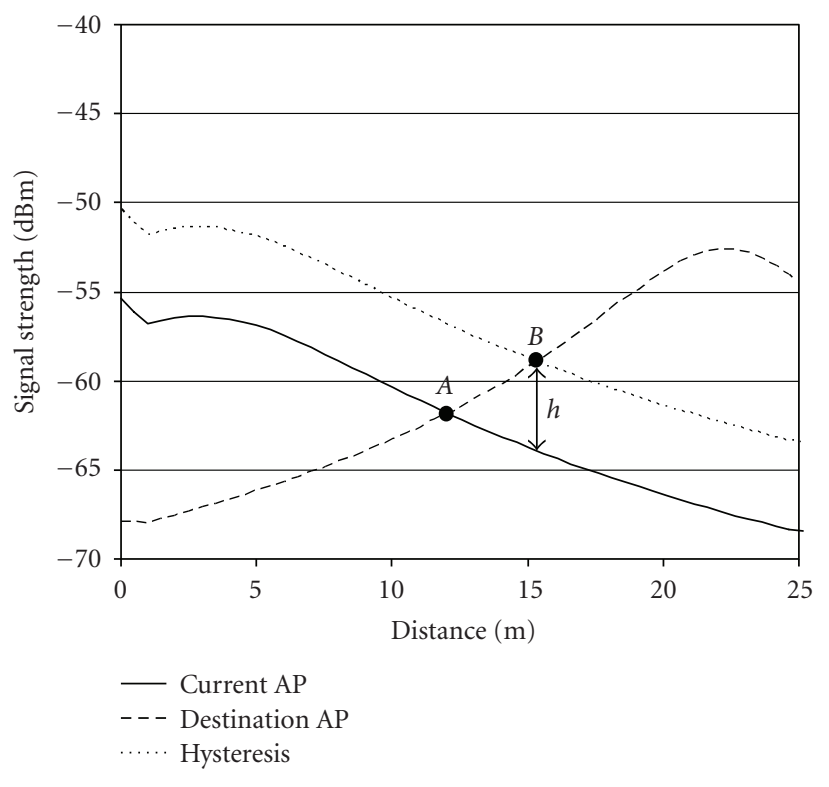

FIGURE 3: Signal strength and handover with hysteresis.

point $B$. This approach absorbs any potential unnecessary handover originated with improvements lower than $h$ on the signal quality.

Following the same approach as in the case of the best server, the probability of having a handover will be

$$
P_{\mathrm{HO}}=\sum_{i=1}^{n} P_{i}\left(s_{i}>s_{0}+h\right) .
$$

This could be expressed in a closed form as

$$
P_{\mathrm{HO}}=\sum_{i=1}^{n} e^{-e^{\left(s_{0}+h-\mu_{i}\right) / \sigma_{i}}} .
$$

In the particular case that same statistics are assumed for the $n+1$ access points in a network, and replacing $s_{0}$ with the expected value $(\mu), h$ could be expressed in a closed form as

$$
h=\sigma \ln \left(\ln \left(\frac{n}{P}\right)\right) .
$$

As can be seen, $h$ depends on the number of access points $(n)$, the probability of having a handover and the standard deviation of the signal strength distribution, but it does not depend on the average signal strength $(\mu)$. From (13), it appears that $h$ depends mainly on the standard deviation of the signal strength, with influence from the number of access points. Notice that the reduction in the probability of having a handover requires an increase of $h$. Similarly, $h$ has to increase in networks with a larger number of access points.

The plain hysteresis approach described has the implicit drawback of producing a certain amount of unnecessary handovers, when the signal strength from the current access point is high, and the ratio $s_{i}>s_{0}+h$ is still possible.

\subsubsection{RSSHThandover}

An improvement over the plain hysteresis handover decision approach is used to reduce the unnecessary handovers, when the signal strength of the current access point is high enough. The improved technique is known as relative signal strength with hysteresis and threshold (RSSHT). This technique is described in [8], and it basically allows a handover of the type $s_{i}>s_{0}+h$ when $s_{0}>T_{C S}$, being $T_{C S}$ the signal threshold. The probability of handover in this case could be expressed as

$$
P_{\mathrm{HO}}= \begin{cases}\sum_{i=1}^{n} P_{i}\left(s_{i}>s_{0}+h\right) & \forall s_{0} \leq T_{C S}, \\ 0 & \forall s_{0}>T_{C S}\end{cases}
$$

or in a closed form:

$$
P_{\mathrm{HO}}= \begin{cases}\sum_{i=1}^{n} e^{-e^{\left(s_{0}-\mu_{i}\right) / \sigma_{i}}} & \forall s_{0} \leq T_{C S}, \\ 0 & \forall s_{0}>T_{C S} .\end{cases}
$$

The selection of the hysteresis margin $h$ could be carried out in the same way as in the plain hysteresis approach.

Using RSSHT, it is possible to reduce the total number of handovers. The probability of having a handover for a given signal strength in the current AP will be influenced by the proper selection of the $T_{C S}$ value. A possibility of selecting a $T_{C S}$ value could be to estimate the signal strength for a given probability of crossing $T_{C S}$ for a given access point. For example, assuming the same power distribution in all the access points, an estimation of the threshold could be

$$
T_{C S}=\mu+\sigma \ln \left(-\ln \left(1-P_{C S}\right)\right),
$$

where $P_{C S}$ (probability of crossing the threshold) could be taken as the same value used for the probability of handover in the estimation of $h$ in (13).

Since a reduction in the number of handovers is possible using RSSHT, this technique is commonly used in commercial products. Nevertheless, there is still a remaining part of handovers that are produced near $T_{C S}$ and are also unnecessary.

\subsubsection{Handover with variable hysteresis}

To increase the performance of conversational video in multicell wireless networks in the presence of handovers, we propose an improvement based on the use of hysteresis techniques with a variable margin. In such a way that when signal strength is high, the probability of a handover taking place is reduced (increase in the $h$ value), and when signal strength is lower, the probability of handover increases (decrease in the $h$ value). This approach will minimize the effect of having unnecessary handovers near $T_{C S}$ using RSSHT.

To obtain a variable hysteresis margin, let us define a lower signal strength reference, called $s_{T}$, this value could be as low as the sensitivity value, but in principle it could be an arbitrary value. If the variable hysteresis margin is defined as 


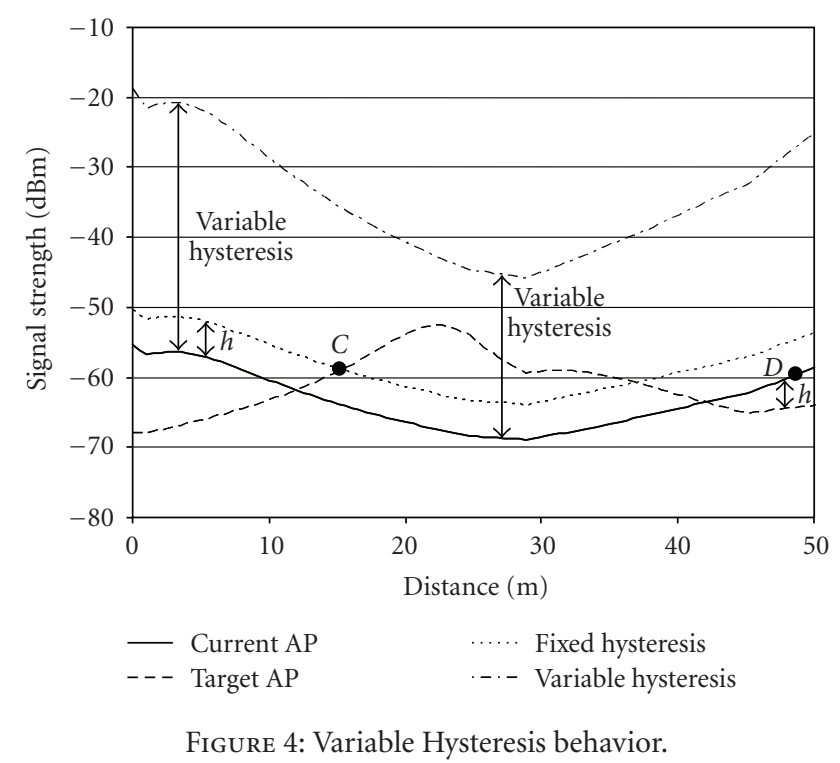

$s_{0}-s_{T}$, where $s_{0}$ is the current $\mathrm{AP}_{0}$ signal strength. Assuming that $\mathrm{AP}_{i}$ is the handover destination mobile node candidate and $s_{i}$ is the power level received from it; handover only will take place if $s_{i}>2 s_{0}-s_{T}$, that is, the higher the signal strength, the higher the hysteresis margin, and therefore the lower the probability of a handover taking place will be.

The behavior of the variable hysteresis is compared to the plain hysteresis in Figure 4 , where it is noticeable that the handovers that took place at point $C$ and $D$ are no longer necessary, since the variable hysteresis line does not cross the power level of the target AP. This effect is possible since the minimum signal strength of the current AP is rather high.

In these conditions, the probability of a handover taking place will be

$$
P_{\mathrm{HO}}=\sum_{i=1}^{n} P_{i}\left(s_{i}>s_{0}+s_{0}-s_{T}\right)=\sum_{i=1}^{n} P_{i}\left(s_{i}>2 s_{0}-s_{T}\right) .
$$

This could be expressed in a closed form as

$$
P_{\mathrm{HO}}=\sum_{i=1}^{n} e^{-e^{\left(2 s_{0}-s_{T}-\mu_{i}\right) / \sigma_{i}}} .
$$

Just as in the case of plain hysteresis, assuming that all signal distributions are similar, the lower reference signal strength could be estimated as

$$
s_{T}=\mu-\sigma \ln \left(\ln \left(\frac{n}{P}\right)\right) .
$$

The lower bound value is also a function of the expected value $\mu$, therefore the selection of this value could depend on how the actual network has been deployed, and could be taken as $s_{T}=\mu-h$, using $h$ as in the plain hysteresis or RSSHT cases.

All of the handover techniques described could produce an abnormal behavior in conditions, where none of the access points available are received with a minimum amount of signal strength. These are typical conditions when outage conditions are produced.

\subsection{Signal outage conditions}

A critical situation will occur when a mobile node such as a handheld device exits, the WLAN, for instance moving outside the coverage area. In this case, the way to keep the communication continuity is whenever possible to carry out a vertical handover to a cellular network.

If $s_{S}$ is the signal level threshold to have acceptable communication in the WLAN, the probability that $\mathrm{AP}_{i}$ is received at the mobile node with a signal below this level will be

$$
P\left(s_{i}<s_{S}\right)=F\left(s_{S}\right)=1-e^{-e^{\left(s_{S}-\mu_{i}\right) / \sigma_{i}}} .
$$

The condition for outage will be met, when all access points are below the threshold value. This condition could be expressed as

$$
P_{\mathrm{VH}}=\prod_{i=0}^{n} P\left(s_{i}<s_{S}\right),
$$

or in a closed form:

$$
P_{\mathrm{VH}}=\prod_{i=0}^{n}\left(1-e^{-e^{\left(s_{S}-\mu_{i}\right) / \sigma_{i}}}\right) .
$$

In these conditions, the mobile node should have decided a vertical handover with certain anticipation. Descriptions of several techniques used for anticipating the vertical handover decision are available in [26]. Proper WLAN deployment designs should maintain the probability of experiencing outages and therefore vertical handovers in the coverage area in low values.

\subsection{Comparison of handover decision approaches}

The different handover approaches described could be compared by making some simplifying assumptions, and evaluating the probability of experiencing a handover for a given current access point signal strength value. Provided that the minimum acceptable signal strength is achievable, the lower the probability of having a handover is, the better the performance is, and therefore the better the associated approach.

Assuming that all access points present the same signal strength statistical behavior, and being consistent with the results of Figures 1 and 2 with $\mu=-70 \mathrm{dBm}, \sigma=10$, and using $h=10 \mathrm{~dB}$ and $s_{T}=-92 \mathrm{dBm}$ for a scenario of four access points, the results for the different approaches are shown in Figure 5.

As can be seen, the probability of a handover taking place for a given signal strength is higher for the case of the best server approach, while minimum for the case of variable hysteresis. The difference between RSSHT and hysteresis appears within the lower handover probability range, caused by the threshold, and in the others both curves are identical and appear to overlap. In these conditions, it performs better than RSSHT, the latter better than hysteresis and hysteresis performs better than the best server. The differences are maintained in the whole range of the power values.

Taking the same values, the probability of signal outage becomes very low $\left(2.6 \cdot 10^{-4}\right)$. 


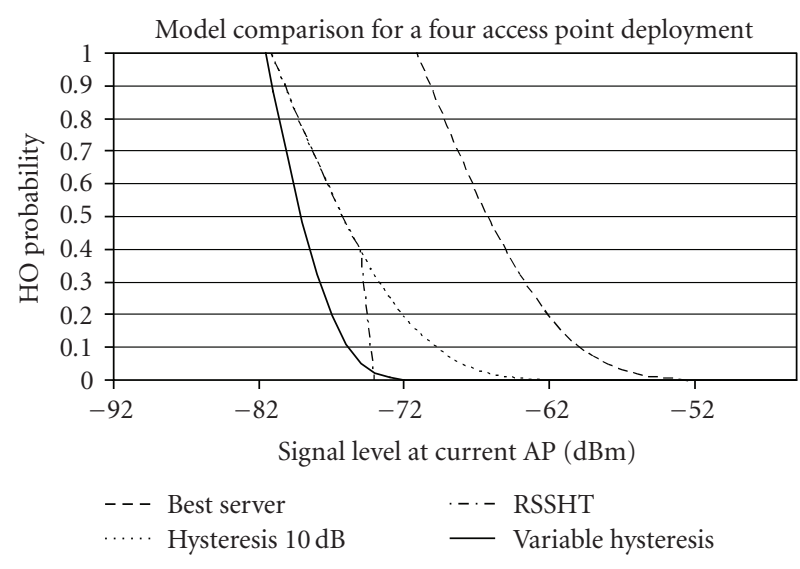

Figure 5: Comparison of the different handover approaches.

\section{CONVERSATIONAL VIDEO PERFORMANCE}

A usual approach to estimating video quality is the peak signal-to-noise ratio (PSNR), or more recently video quality rating (VRQ) both are usually estimated from the mean square error (MSE) of the video frames after the impairments (e.g., packet loss) with respect to the original video frames $[27,28]$. From these values, there is some correlation to the video mean opinion score (MOS), unfortunately, the relationship between packet loss and MSE is not straightforward, since not all packets conveyed through the wireless network have the same significance. Alternatively, a relatively simpler quality indicator is proposed in [14]. This indicator is the effective frame rate, which is introduced and discussed in later sections of this paper. This paper also proposes a model to characterize the impact of packet losses on the effective frame rate of the video sequence.

As packet losses occur in the wireless network, video frames are damaged; making some of them unusable, and therefore the total frame rate is reduced. Video quality will be acceptable, if the expected frame rate of the video conversations is kept above certain value.

The consequence of a packet loss in a generic video sequence depends on the particular location of the erroneous packet in the compressed video sequence. The reason for this is related to how compressed video is transmitted through the IP protocol. The plain video source frames are compressed to form a new sequence of compressed video frames or slices. The new sequence could be, depending on the H.264 service profile applied, made up of three types of frames: I (Intra) that transports the content of a complete frame with lower compression ratio, $P$ (Predictive) that transports basic information on an prediction of the next frame based on movement estimators, and $B$ (Bidirectional) that transports the difference between the preceding and the following frame. This sequence of slices is grouped into the so-called group of pictures (GoPs) or groups of video $(\mathrm{GoV})$ objects depending on the standard. The GoV could adopt many forms and structures, but for our analysis, we assume a typical configuration of the form IPBBPBBPBBPBBPBB. This means that every 16 frames there is an Intra followed by Predictive and Bidirectional frames. IP video packets are built from pieces of the aforementioned frame types and delivered to the network. If a packet error has been produced in a packet belonging to an Intra frame, the result is different from the same error produced in a packet belonging to a Predictive or Bidirectional frame.

There are some characteristics that are applicable to the case of conversational video, and in particular to portable conversational video, that are not necessarily applicable to other video services like IPTV or video streaming. The first important characteristic is the low-speed and low-resolution formats (common intermediate format, CIF, or quarter CIF, QCIF), that in turn produce a very low number of packets per frame, especially, if protocol efficiency is taken into account by increasing the average packet size. In these conditions, a single packet could convey a substantial part of a video frame. The second important characteristic comes from the portability and low consumption requirement at the receiving end that in turn requires a lighter processing load to save battery life. The combination of the two aforementioned characteristics makes packet losses impact greatly on the frame integrity and concealment becomes very restrictive. In conversational video, it could be better for instance to maintain a clear fixed image of the other speaker on the screen, than to try error compensation at the risk of severe image distortions and artifacts. Following these characteristics, every time that a packet is lost in a frame, the complete frame becomes unusable, and some actions could be taken at the decoder end to mitigate the effect, such as freezing or copying frames, but the effective frame rate has been reduced and has to accept some form of video quality degradation.

It is possible to obtain a simple video quality estimator based on the effective frame rate resulting from the packet losses due to the handover effect, as introduced in [14]. Although this could not be generalized for all types of IP video, in the case of conversational video, this indicator presents advantages over the use of PSNR: allows simple relationship between the packet loss and objective quality, and intuitively represents the behavior of conversational video over a wireless network.

\subsection{Handover impact on video quality}

In the present analysis, it is considered that the handover duration $T_{s}$ is longer than a video frame. Typical handover durations can be found in [15]. When the handover duration affects several slices, the video stream can only be displayed once an I frame is received, as shown in Figure 6. If $T_{s}$ is the GoV and $T_{h}$ is the handover duration, two cases are possible when $T_{h}<T_{s}$ and the handover event involves slices from two GoV or when $T_{h}>T_{s}$. For the rest of the analysis, it is considered that $T_{h}<T_{s}$, (e.g., $T_{h}<1000$ milliseconds) and therefore the number of frames lost are always less than two GoVs.

Following this principle, it is possible to estimate the expected number of frames lost due to a handover. Provided that several frames are lost in a handover, the video stream will need to wait until the next I slice, and therefore the 


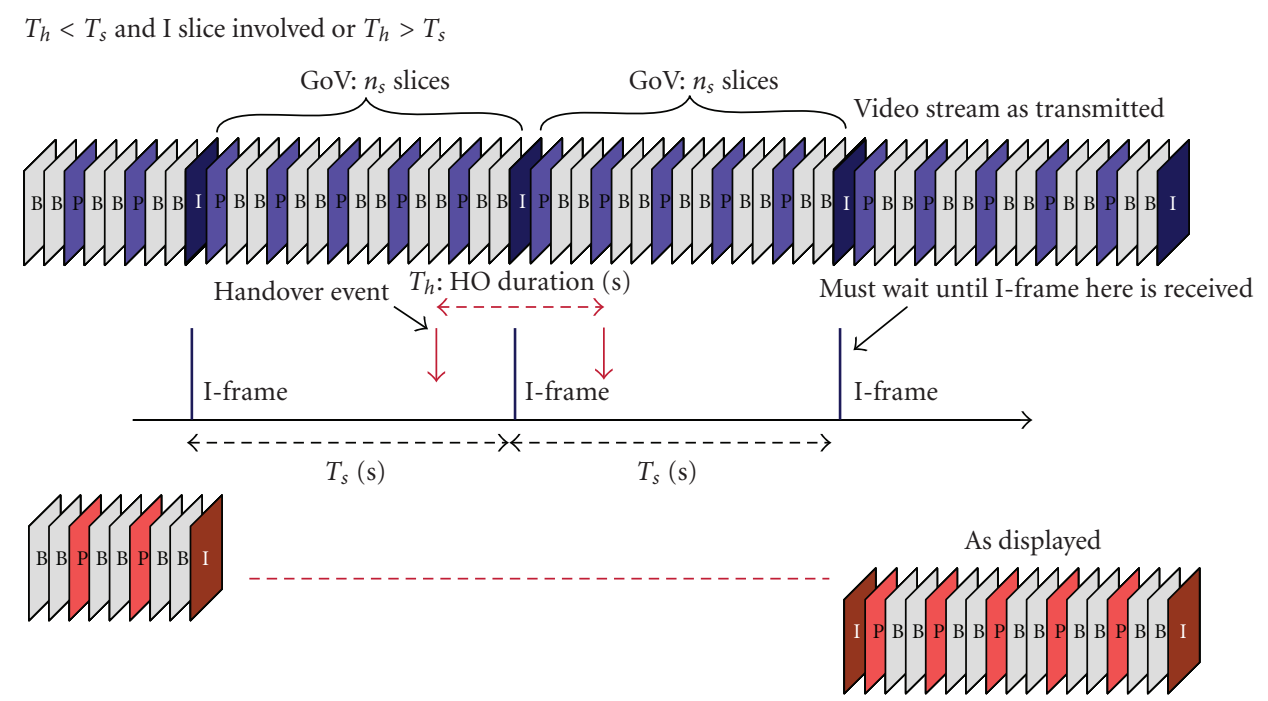

FIgURE 6: Handover effect on displayed video stream.

expected number of frames lost will depend in the position of the handover initiation in the GoV, which can be expressed as

$$
E_{1}=\frac{1}{n_{s}} \sum_{i=0}^{n_{s}-1}\left(n_{s}-i\right) .
$$

Depending on the handover duration, it is possible that the handover takes place at the end of one GoV, taking the first slices of the next, which makes GoV unusable. This effect produces an additional expected number of lost frames, which could be expressed as

$$
E_{2}=\frac{1}{n_{s}} \sum_{j=1}^{n_{h}} n_{s}
$$

where $n_{h}$ is the handover duration expressed in number of slices, that could be calculated from

$$
n_{h}=\operatorname{ceil}\left(T_{h} f_{0}\right)
$$

where $T_{h}$ is the handover duration, and $f_{0}$ is the video frame rate.

The expected number of frames lost in the video sequence due to a handover will be

$$
E=E_{1}+E_{2}=\frac{n_{s}}{2}+n_{h}-1
$$

The performance of video in terms of frame rate in the presence of handovers could be expressed as follows:

$$
f=f_{0}\left(1-E \cdot P_{\mathrm{HO}}\right) \text {. }
$$

The performance will depend on the handover technique used. If the RSSHT technique is used, the resulting frame rate obtained bycombining (15), (26), and (27) will be

$$
f=f_{0}\left(1-\left(\frac{n_{s}}{2}+n_{h}-1\right) \sum_{i=1}^{n} e^{-e^{\left(s_{0}-\mu_{i}\right) / \sigma_{i}}}\right) \quad \forall s_{0} \leq T_{C S}
$$

If variable hysteresis is used, by combining (18), (26), and (27) the resulting frame rate will come from

$$
f=f_{0}\left(1-\left(\frac{n_{s}}{2}+n_{h}-1\right) \sum_{i=1}^{n} e^{-e^{\left(2 s_{0}-s_{T}-\mu_{i}\right) / \sigma_{i}}}\right) .
$$

The previous approach is also applicable to the case of signal outage conditions, that is, none of the access points is received above the sensitivity threshold. In this case, (27) becomes

$$
f=f_{0}\left(1-E \cdot\left(P_{\mathrm{HO}}+P_{\mathrm{VH}}\right)\right) .
$$

Since signal outage is produced when no handover is possible, in the case of signal outage, (30) becomes

$$
f=f_{0}\left(1-\left(\frac{n_{s}}{2}+n_{h}-1\right) \prod_{i=0}^{n}\left(1-e^{-e^{\left(s_{S}-\mu_{i}\right) / \sigma_{i}}}\right)\right) .
$$

Equation (31) is valid for the cases in which the signal outage duration is longer than one video frame, which is the typical case. This effect is mainly related to the radio network design and could have very low impact.

In Figures 7 and 8, the case of four cells is shown. According to the signal levels, there is certain probability of experiencing a handover, and thus undergoing a reduction in the frame rate. The example covers several handover durations. If the acceptability limit is considered to be 5 frames/second [29], quality outage can be evaluated as a function of the signal strength coming from the radio network design.

It must be noted that in Figure 7 outage starts at $-76 \mathrm{dBm}$, while in Figure 8 it appears at $-79 \mathrm{dBm}$, which is a $3 \mathrm{~dB}$ improvement.

\subsection{Solution to improve handover impact on video quality}

The conversational video degradation due to the handover processes taking place in a wireless network can be addressed 


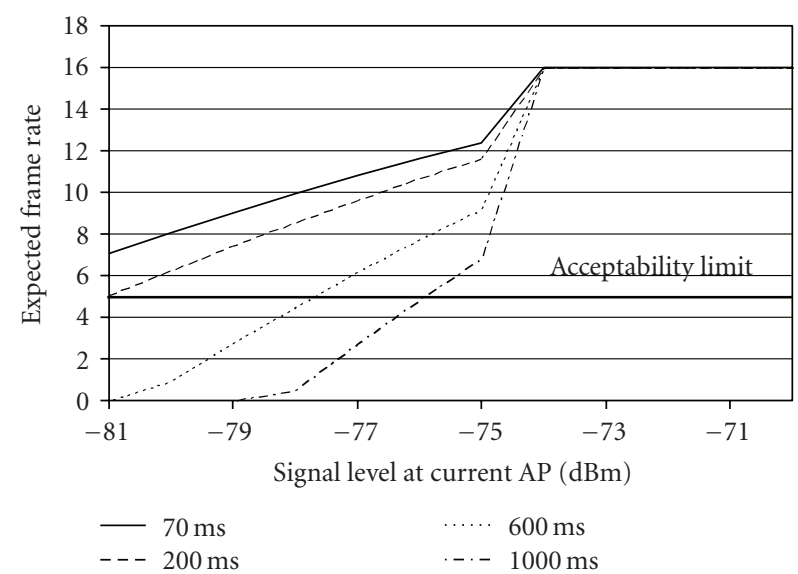

FIgURE 7: Frame rate as a function of the signal quality according to handover probability in multicell networks in the case of RSSHT.

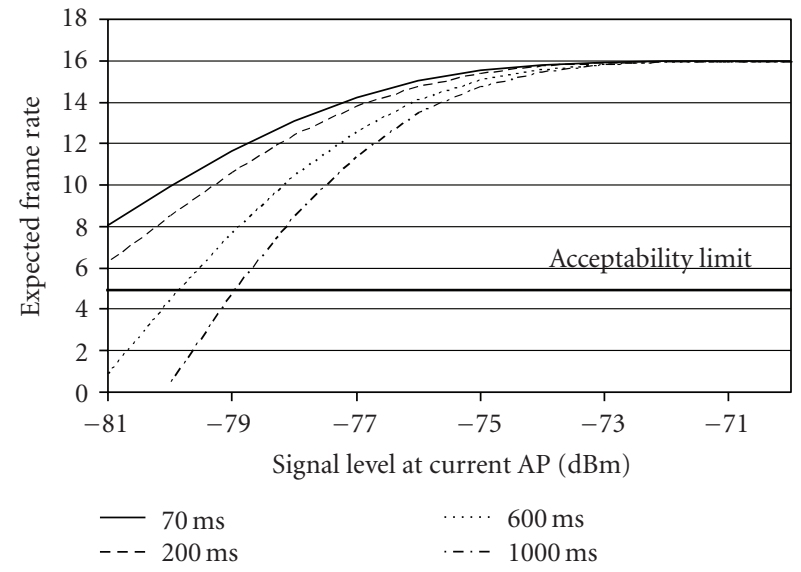

FIGURE 8: Frame rate as a function of the signal quality according to handover probability in multicell networks in the case of variable hysteresis.

from at least two general perspectives: video processing to mitigate whatever effect is taking place in the transmission media, For example, degradations due to channel, handovers, call drops, and so forth, and wireless processing carried out in the wireless part. In the previous section, the variable hysteresis handover technique has been proposed in order to minimize the handover probability, in this section the solution proposed focuses on the video part.

In fact, a technique that could improve the video quality caused by handover will be to reduce the GoV objects lost due to frame interdependence. This will be possible by forcing a reset of the GoV to generate an I frame, breaking off the natural sequence of frames IPBBPBBPBBPBBPBB and reducing the time between I frames. An example is shown in Figure 9.

There are, at least, two possible techniques to implement the proposed reset. First, to take advantage of channel reciprocity for conversational video, which means that downand uplinks channel behaviors are closely related, so the transmitter end can detect a handover event automatically as well as generating the I frame automatically when required. Another technique will be to introduce a feedback from receiver to transmitter through a real-time control protocol.

In these conditions, the new expected value of frames lost in the process will consist of at least one frame lost to detect the event and an additional frame to reset the GoV. On top of these 2 frames, the total duration of the handover will need to be translated in a frame count.

Taking as example the RSSHT case, (28) will become

$$
f=f_{0}\left(1-\left(n_{h}+2\right) \sum_{i=1}^{n} e^{-e^{\left(s_{0}-\mu_{i}\right) / \sigma_{i}}}\right) \quad \forall s_{0} \leq T_{C S} .
$$

As a result, there will be a considerable statistical improvement in the case of short handovers, while this improvement is smaller in the typical case of longer handovers; in any case, it also depends on the relative distance between the handover end event and the next Intra-frame. Figure 10 compares the different approaches discussed in terms of expected frame rate as a function of the average signal level received at the AP. The expected frame rate represents the effective frame rate resulting from the packet losses due to the handover effect. Handover duration is 1000 milliseconds in Figure 10. The shape of the curves is strongly influenced by the handover technique used. Variable hysteresis produces softer shape, while RSSHT produces a somehow abrupt behavior. As can be seen, the greater improvement is achieved with the variable hysteresis approach although some additional improvement is also possible combining both variable hysteresis and Intra reset. Most of the gain is produced by the use of variable hysteresis, as will be confirmed by simulations.

\section{SCENARIO SIMULATION}

To illustrate the principles shown in the above sections, an example has been selected as shown in Figure 11. The scenario corresponds to an office environment of $20.2 \times 35.5$ meters covered with four access points represented with the color shapes inside the layout, each color associated to a frequency channel. The walls have been modeled to introduce attenuation in the signal propagation and edge diffraction. The position of the access points has been found with an automatic process to optimize the coverage and capacity simultaneously. The dashed line represents a trajectory of a mobile node at $4 \mathrm{Km} / \mathrm{h}$ in the scenario, covering a total distance of $190 \mathrm{~m}$.

The handover performance could be assessed for the different conditions described in the previous sections. The simulations consist of the use of RSSHT and of variable hysteresis techniques with different handover durations through the trajectory and comparing the results. The power histograms of the four access points through the trajectory are shown in Figure 12. Several iterations are also carried out to analyze the influence and sensitivity of the design parameters on the final performance. 

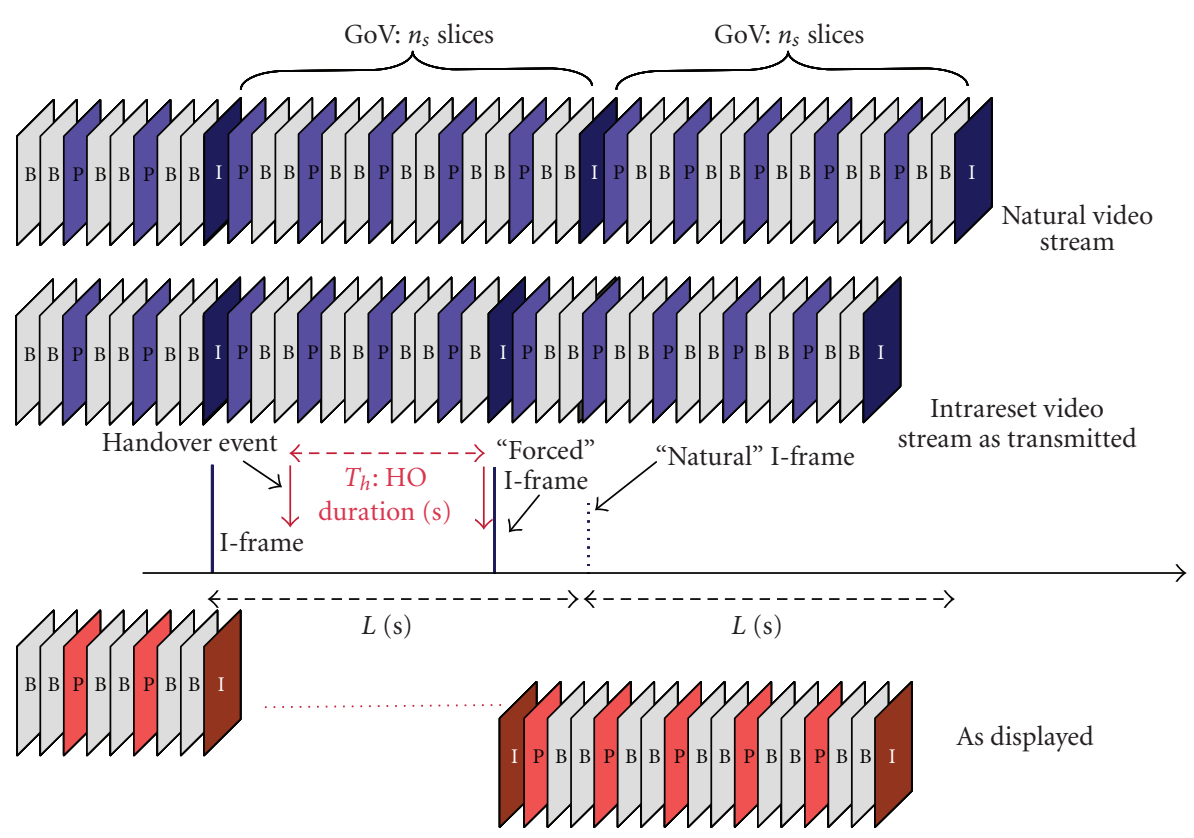

FIGURE 9: Handover effect on displayed video stream with Intra reset.

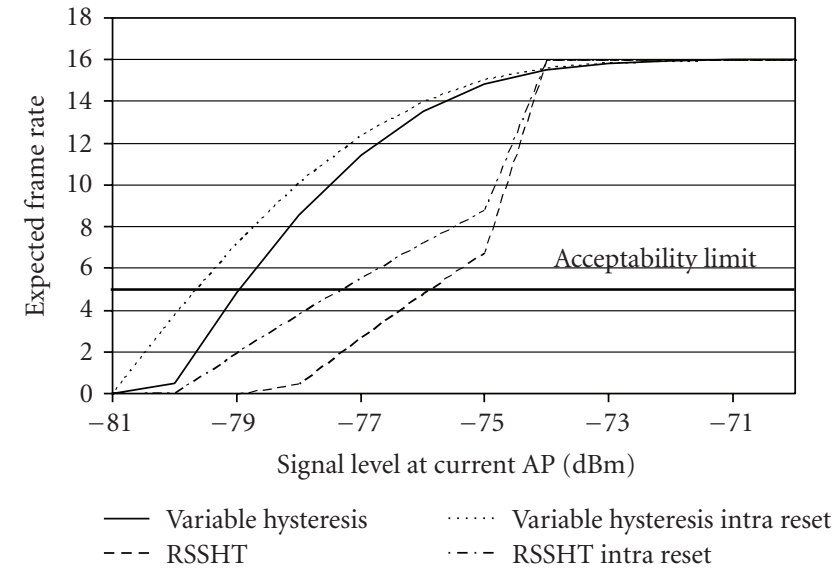

FIGURE 10: Frame rate performance for 1 second handovers using the different techniques.

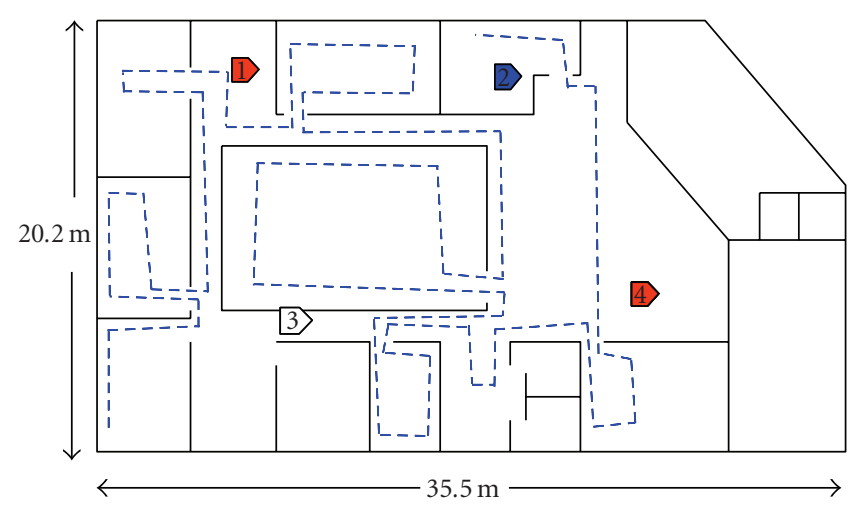

Figure 11: Simulation scenario.

\subsection{RSSHT handover parameter simulation}

The use of a threshold above, where no handover action takes place, produces some improvement in the total number of handover events, even without using hysteresis, this is due to the simple fact that some of the best server handovers are taking place at relatively high signal strengths. Figure 13 represents handover events as a function of time to move through the trajectory by the mobile node at a pedestrian speed. As shown in Figure 13, the total number of handovers for the $3 \mathrm{~dB}$ margin is reduced from 25 to 17 , and with $7 \mathrm{~dB}$ the reduction is from 17 to 12 . An additional margin increases to $16 \mathrm{~dB}$, produces a further reduction in the total handover events down to 6 , but at the expense of having the lowest signal strength below the system sensitivity. Just as in the case of plain hysteresis, in this case the distribution of handover events also depends on $h$, and the increase in $h$ is stripping handovers from the registry, and slightly changing their position.

The impact of the hysteresis margin on the number of handover events using threshold is shown in Figure 14. The behavior is monotonic down to $19 \mathrm{~dB}$ where 4 handovers are produced; an additional increase in the margin produces no improvement in the handover count.

Although the number of handovers decrease monotonically as the hysteresis margin increases, it is essential to analyze the impact on the minimum signal level. The effect of the hysteresis margin on the minimum signal strength value is shown in Figure 15. The use of a threshold has less influence on the minimum signal strength observed. Hysteresis greater than $9 \mathrm{~dB}$ produces minimum signal strength in the range of the system sensitivity, therefore a value lower than $9 \mathrm{~dB}$ is the best choice for the $h$ value. Let 


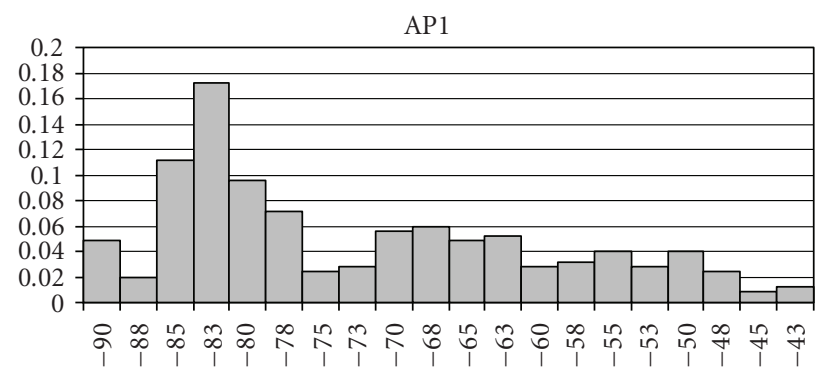

(a)

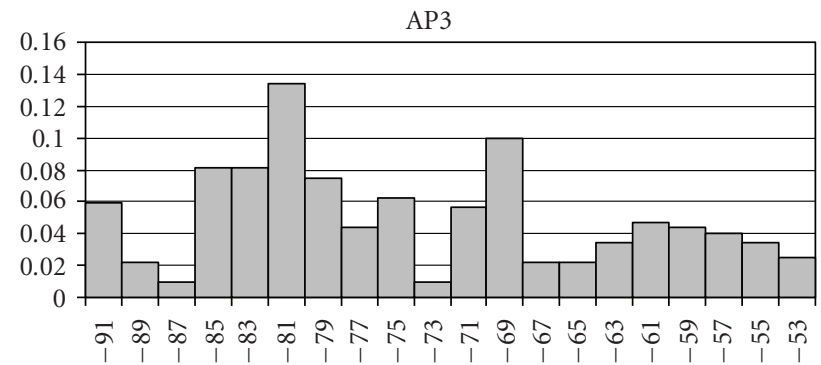

(c)

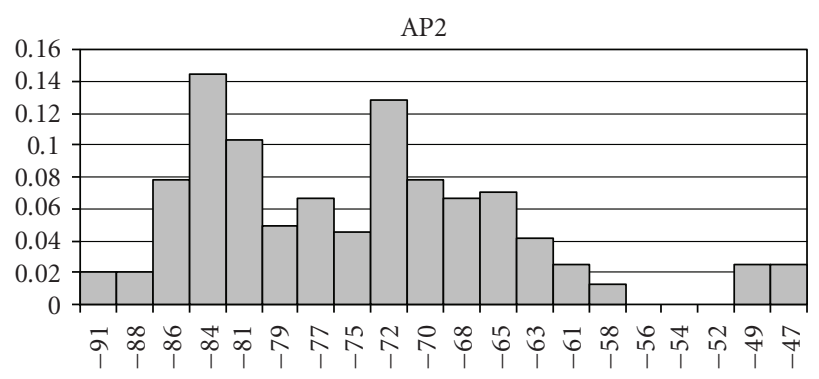

(b)

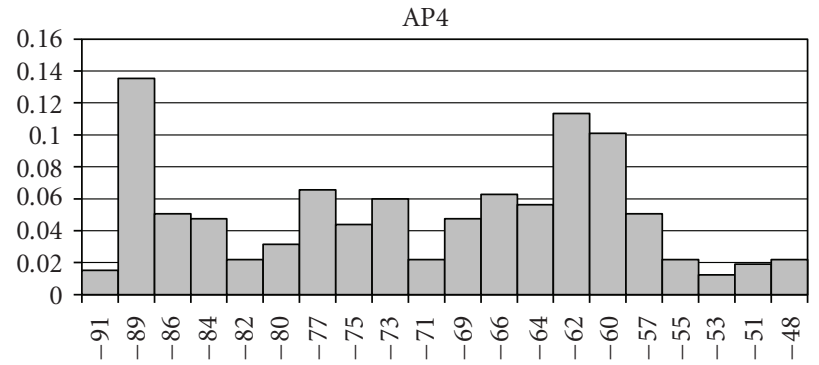

(d)

FIgURE 12: Power histograms for the four APs through the trajectory.

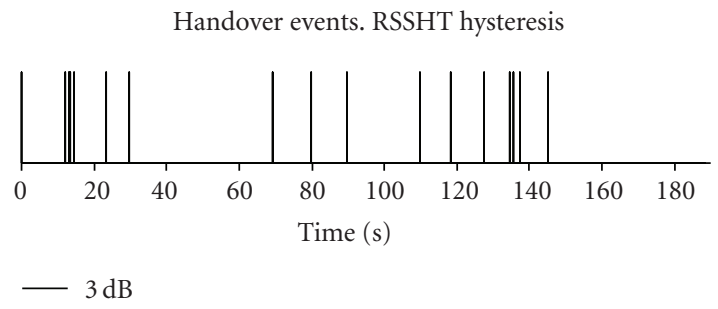

(a)
Handover events. RSSHT hysteresis

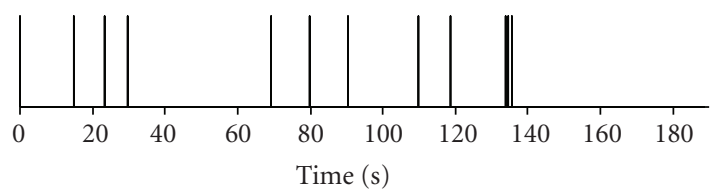

$-7 \mathrm{~dB}$

(b)

Handover events. RSSHT hysteresis

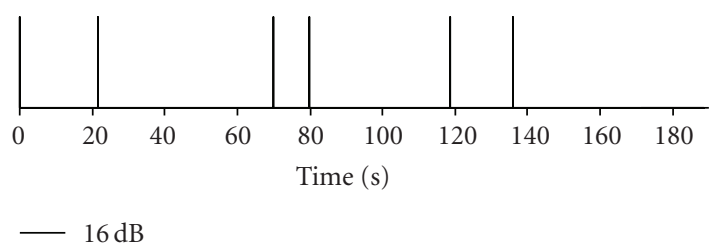

(c)

Figure 13: Handover events using RSSHT.

us take $7 \mathrm{~dB}$, since it is producing a minimum signal in the range of $-86 \mathrm{dBm}$.

Summarizing, the $h$ value has to be as small as possible to keep the minimum signal level as high as possible, but at the same time $h$ has to be as large as possible to reduce the total handover count. The tradeoff selected has been $h=7 \mathrm{~dB}$.

\subsection{Variable hysteresis simulation}

One of the advantages of the variable hysteresis techniques is that there is no need for parameter tuning. The only parameter is the lower boundary that can be the system sensitivity threshold that is usually $-92 \mathrm{dBm}$ for typical IEEE802.11 WLAN.

Figure 16 shows the results obtained using variable hysteresis in the reference scenario. As can be seen, the number of handovers has been reduced from 12 (optimal RSSHT with hysteresis of $7 \mathrm{~dB}$ ) to 9 keeping the same value of minimum signal strength in the trajectory.

In summary, variable hysteresis produces additional reduction in the unnecessary handover count with respect to the RSSHT. 


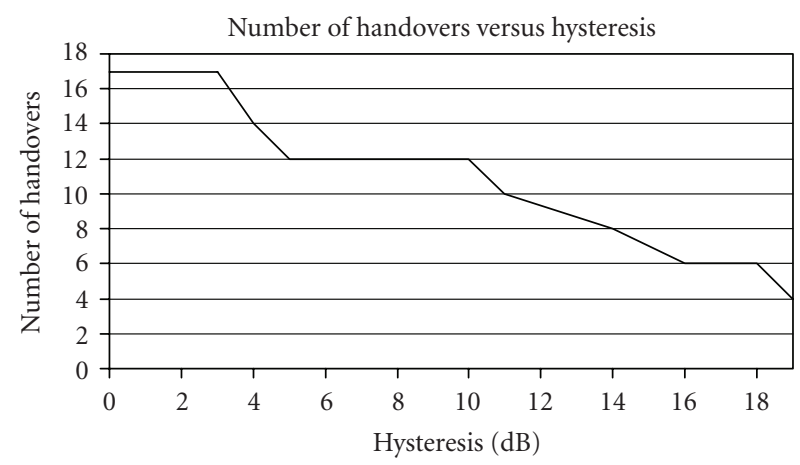

FIGURE 14: Handover events as a function of the hysteresis margin using $T_{C S}=-80 \mathrm{dBm}$.

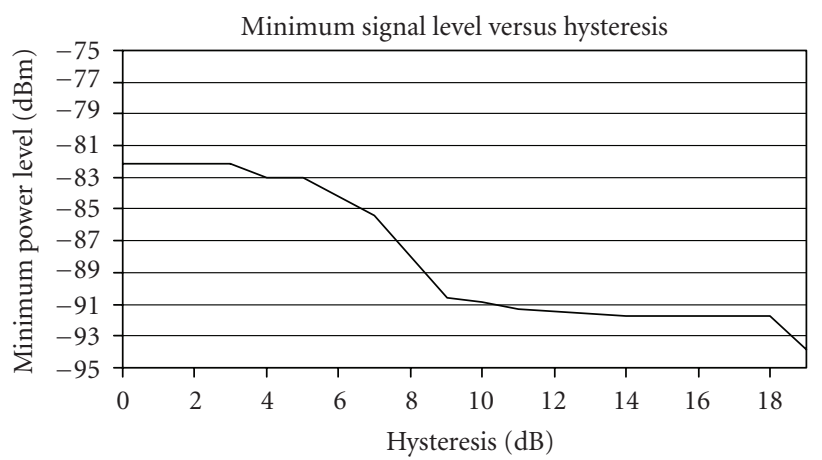

FIGURE 15: Minimum signal strength as a function of the hysteresis margin for $T_{C S}=-75 \mathrm{dBm}$.

\section{SCENARIO PERFORMANCE RESULTS}

Taking the RSSHT handover technique with the parameter setting from the previous sections, it is possible to analyze the impact of the handovers in the conversational video quality.

The approach followed has been to simulate the video performance for different handover durations in the scenario shown in Figure 11.

Assuming that the total duration of the trajectory is 171 seconds, corresponding to a relative low moving speed, the average frame rate of the conversational video obtained in different handover durations is shown in Figure 17.

The fluctuations of the frame rate are due to the frames lost in the handover process. Depending of the duration of the handovers and the frequency of the occurrence, the frame rate can be reduced down to values that are not acceptable for the user. If the generally accepted value of 5 frames/second [29] is taken as figure of merit for video acceptability, it is possible to see how the frame rate evolves and where it will cross the limit. Figure 17 presents this circumstances starting at 134 seconds for all handover durations. This effect is due to the accumulation of handovers starting at this point of the trajectory, as can be seen in Figure 13, not allowing the video frame rate to recover.

If the frame rate drops to 5 frames/second or below, it is considered as producing an outage, and the outage ends once

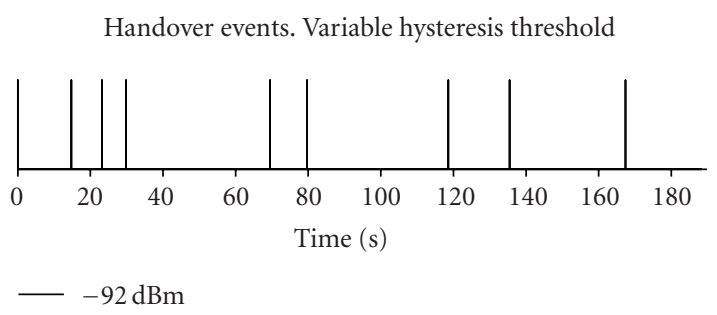

FIGURE 16: Handover using variable hysteresis.

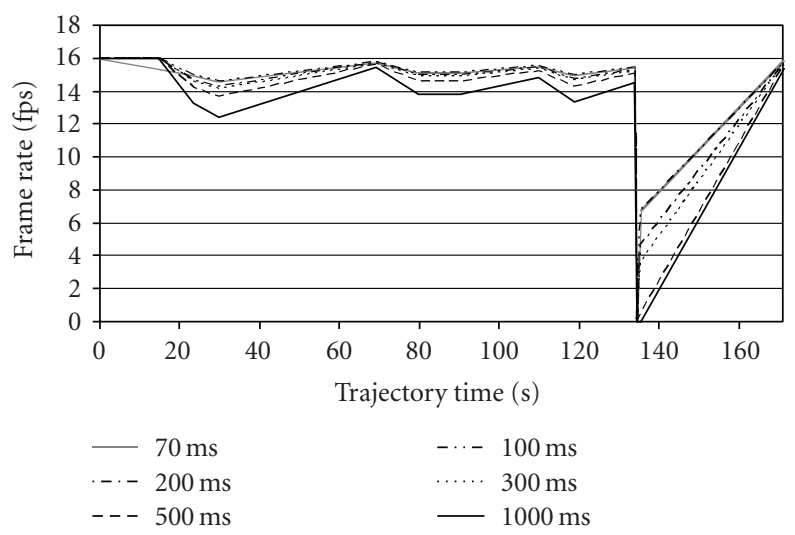

Figure 17: Resulting frame rate as a function of the handover duration using RSSHT.

the frame rate raises again above 5 frames/second. Using this principle, the duration of the quality outages can be estimated.

By repeating the process for different handover durations, it is possible to assess the influence of the handover duration on the quality outages.

If $\mathrm{GoV}$ reset is applied, the results are significantly improved as shown in Figure 18.

Figure 19 shows the effect of the handover duration on the outage in the simulation scenario for normal RSSHT and RSSHT with Intra reset. As can be seen, the quality outage grows monotonically with the handover duration as expected.

In the present simulation, it is noticeable that handover durations longer than 600 milliseconds produce a relatively flat quality outage in RSSHT, this means that above 600 milliseconds the outage of $7.5 \%$ of time is obtained no matter how long the handover is (up to the simulated value of 1000 milliseconds). To explain this behavior, it is necessary to revisit Figure 13. It is clearly visible that an accumulation of handovers can be seen at the end of the trajectory. These handover events are due to a sequence of abrupt signal fluctuations received from two of the access points (AP3 and AP4) in the scenario. This effect is partially mitigated by using the GoV reset in the low range of the handover duration, as it can be expected.

Using the variable hysteresis technique, the average duration of the frame rate along the trajectory is shown in Figure 20. The frame rate values obtained are above 12 


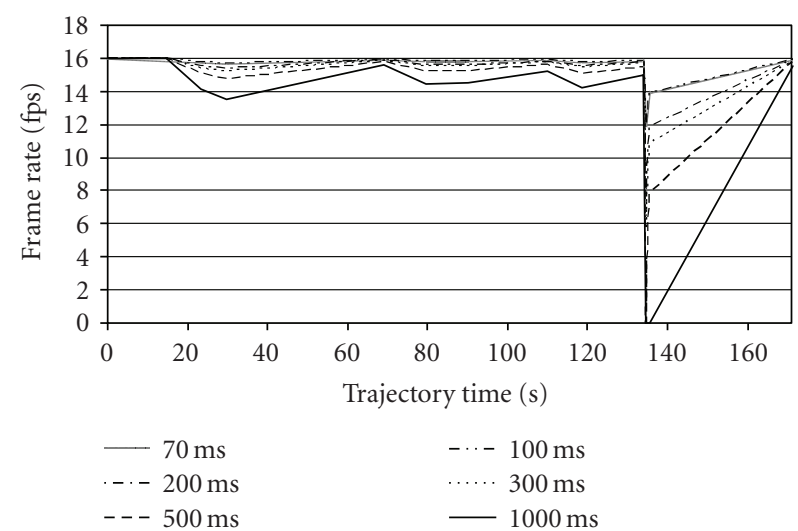

FIgURE 18: Resulting frame rate as a function of the handover duration using RSSHT with GoV reset.

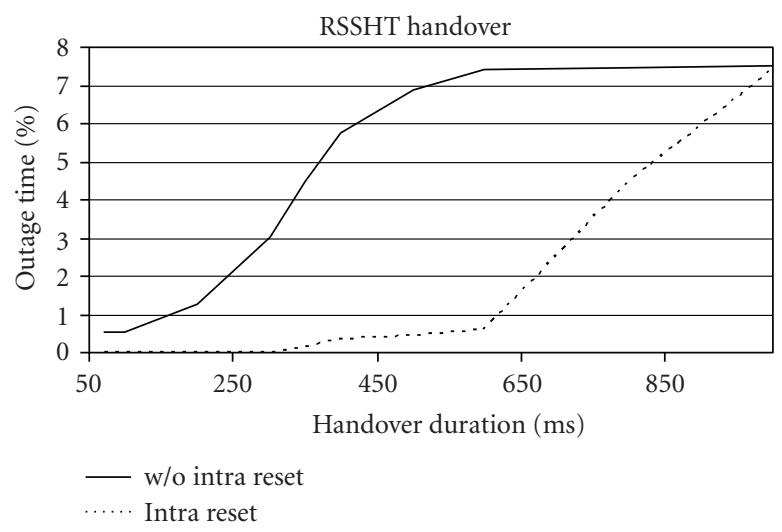

FIGURE 19: Quality outage time as a function of the handover duration.

frames per second in all cases which is way above the acceptability limit. The average frame rate experiences rather low reductions thanks to the positions of the handover events that are rather spread along the trajectory

A final comparison has been performed simulating PSNR as indicator to validate the results obtained. To use PSNR as quality indicator, it is necessary to use a known video sequence and for that purposes the "Foreman" sequence has been used. For the simulations, the following parameters have been considered: QCIF test sequence "Foreman" (25 frames/second, 300 frames), mobile user at $4 \mathrm{Km} / \mathrm{h}$, compressed bit rate at $32 \mathrm{Kbps}$ and average handover duration of 1000 milliseconds. Since "Foreman" is shorter than the duration of the simulated trajectory, the video sequence has been concatenated several times. The technique used for received frame lost concealment is the frame copying, that is, an interval of lost frames is replaced by the last usable frame received.

The results obtained repeating the process for the different techniques, and averaging between handover events are shown in Figure 21. Average PSNR at $32 \mathrm{Kbps}$ bit rate without handover is $31 \mathrm{~dB}$ [30] and a $25 \mathrm{~dB}$ PSNR acceptability limit is assumed. Figure 21 shows similar results as for the case of frame rate. In fact, the outage appears at

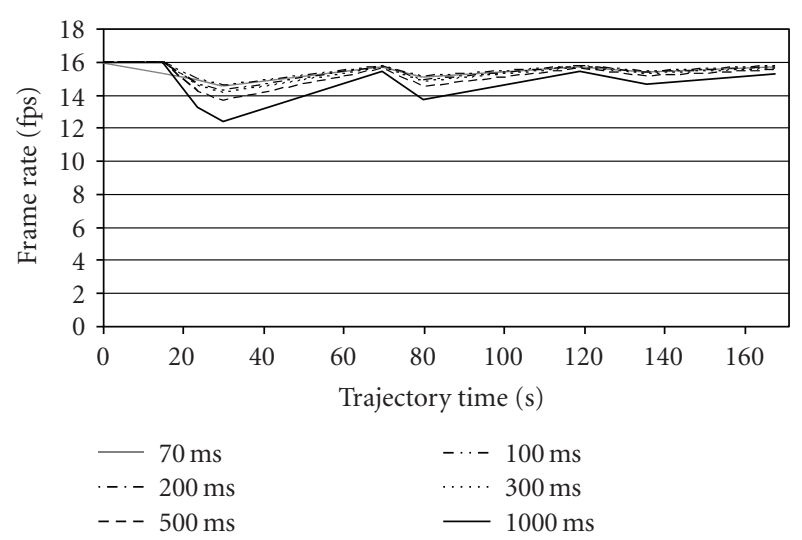

FIgURE 20: Resulting frame rate as a function of the handover duration using variable hysteresis.

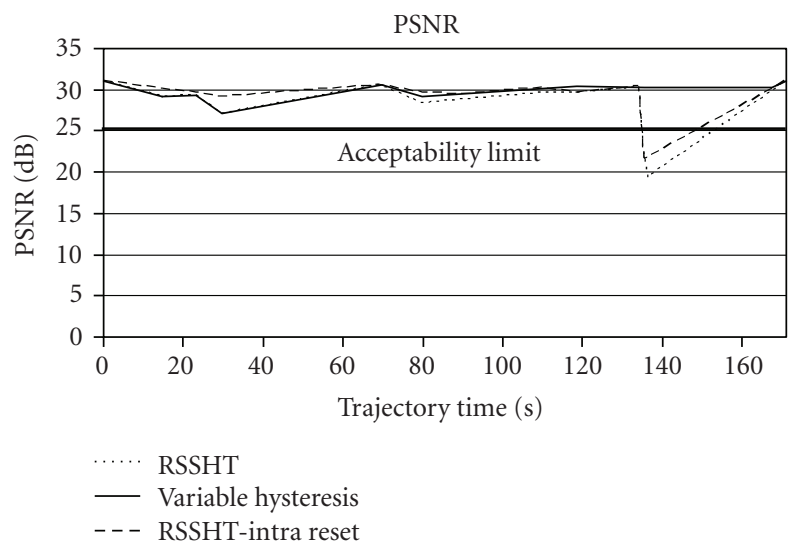

Figure 21: Simulated PSNR using Foreman video sequence.

the end of the path with both RSSHT techniques, with and without Intra reset, however variable hysteresis solves this problem.

\section{CONCLUSIONS AND FUTURE WORK}

The effect of horizontal handover on the conversational video performance in multicell WLAN has been analyzed, and a simple method to predict the impact on the video performance in terms of frame rate has been proposed. The RSSHT handover decision approach, which is a commonly used technique, has been taken as reference for the video performance, and the parameter settings have been discussed. A new technique called variable hysteresis has been proposed and shows a substantial improvement in conversational video performance. A video transport improvement based on the GoV reset after a handover event has also been considered to enhance video quality. The proposed analysis method has been applied to a real scenario by simulating a range of handover durations. The simulations show how video frame rate fluctuates along the trajectory due to the handovers. The scenario has also shown how an accumulation of handovers could produce outages of video quality using RSSHT. The quality outage periods 
grow with the handover duration. The improvement coming from the video stream modification has been compared with those coming from variable hysteresis handover decision technique: the new technique proposed at this paper. The results show that improvements in the handover process reducing unnecessary handovers produces higher quality gain than the video transport processing.

Subsequent research steps will be, first, a more profound performance analysis and simulation of the variable hystereris handover decision mechanisms to further reduce the handover count in multicell deployments to increase the quality. Second, to introduce new video quality indicators that could also be applicable to video streaming and mobile TV.

\section{ACKNOWLEDGMENTS}

The authors are thankful for the support of the Spanish Ministry of Education and Science within the framework of the TEC2005-07010-C02-01/TCM and the TSI2005-07306-C0201/CASERTEL-NGN projects chair in Madrid Polytechnic University. The authors are also thankful for the support of CELTIC Project Easy Wireless II.

\section{REFERENCES}

[1] S. Kashihara and Y. Oie, "Handover management based on the number of retries for VoIP on WLANs," in Proceedings of the 61st IEEE Vehicular Technology Conference (VTC'05), vol. 4, pp. 2201-2206, Stockholm, Sweden, May-June 2005.

[2] Draft Standard for Information Technology Telecommunications and informationexchange between systems-Local and metropolitan area networks-Specific requirementspart 11: Wireless LAN Medium AccessControl (MAC) and Physical Layer (PHY) specifications_-Amendment 2: Fast BSS Transition. Active Unapproved Draft.

[3] A. Mishra, M. Shin, and W. Arbaugh, "An empirical analysis of the IEEE 802.11 MAC layer handoff process," Computer Communication Review, vol. 33, no. 2, pp. 93-102, 2003.

[4] M. Zonoozi and P. Dassanayake, "Handover delay and hysteresis margin in microcells and macrocells," in Proceedings of the 8th IEEE International Symposium on Personal, Indoor and Mobile Radio Communications (PIMRC'97), vol. 2, pp. 396400, Helsinki, Finland, September 1997.

[5] M. Gudmundson, "Analysis of handover algorithms [microcellular radio]," in Proceedings of the 41st IEEE Vehicular Technology Conference (VTC'91), pp. 537-542, St. Louis, Mo, USA, May 1991.

[6] A. Murase, I. Symington, and E. Green, "Handover criterion for macro and microcellular systems," in Proceedings of the 41st IEEE Vehicular Technology Conference (VTC '91), pp. 524-530, St. Louis, Mo, USA, May 1991.

[7] G. E. Corazza, D. Giancristofaro, and F. Santucci, "Characterization of handover initialization in cellular mobile radio networks," in Proceedings of the 44th IEEE Vehicular Technology Conference (VTC'94), vol. 3, pp. 1869-1872, Stockholm, Sweden, June 1994.

[8] F. González, J. A. Pérez, and V. H. Zárate, "HAMS: layer 2 handoff accurate measurement strategy in WLANs 802.11," in Proceedings of the 1st International Workshop on Wireless Network Measurements (WiNMee '05), pp. 1-7, Trentino, Italy, April 2005.
[9] I. Marsh and B. Grönvall, "Performance evaluation of voice handovers in real 802.11 networks," in Proceedings of the 2nd International Workshop on Wireless Network Measurements (WiNMee '06), Boston, Mass, USA, April 2006.

[10] A. F. Duran, E. C. del Pliego, and J. I. Alonso, "Effects of handover on voice quality in wireless convergent networks," in Proceedings of the IEEE Radio and Wireless Symposium (RWS '07), pp. 23-26, Long Beach, Calif, USA, January 2007.

[11] ITU-T Recommendation H.264, "Infrastructure of audiovisual services-Coding of moving video. Advanced video coding for generic audiovisual services," September 2005.

[12] E. Masala, C. F. Chiasserini, M. Meo, and J. C. De Martin, "Real-time transmission of H.264 video over 802.11-based wireless ad hoc networks," in Proceedings of the Workshop on DSP in Vehicular and Mobile Systems, pp. 193-207, Springer, Nagoya, Japan, April 2003.

[13] D. Singh, S. Hoh, A. L. Y. Low, F. L. Lim, S. L. Ng, and J. L. Tan, "Qualitative study of intelligent access point handover in WLAN systems," in Proceedings of the 10th IEEE/IFIP Network Operations and Management Symposium (NOMS '06), pp. 14, Vancouver, BC, Canada, April 2006.

[14] N. Feamster and H. Balakrishnan, "Packet loss recovery for streaming video," in Proceedings of the 12th International Packet Video Workshop, pp. 1-11, Pittsburgh, Pa, USA, April 2002.

[15] J. Montavont, N. Montavont, and T. Noel, "Enhanced schemes for L2 handover in IEEE 802.11 networks and their evaluations," in Proceedings of the 16th IEEE International Symposium on Personal, Indoor and Mobile Radio Communications (PIMRC '05), vol. 3, pp. 1429-1434, Berlin, Germany, September 2005.

[16] P. Dassanayake, "Dynamic adjustment of propagation dependant parameters in handover algorithms," in Proceedings of the 44th IEEE Vehicular Technology Conference (VTC'94), vol. 1, pp. 73-76, Stockholm, Sweden, June 1994.

[17] N. Zhang and J. M. Holtzman, "Analysis of handoff algorithms using both absolute and relative measurements," in Proceedings of the 44th IEEE Vehicular Technology Conference (VTC'94), vol. 1, pp. 82-86, Stockholm, Sweden, June 1994.

[18] T. K. Sarkar, Z. Ji, K. Kim, A. Medouri, and M. SalazarPalma, "A survey of various propagation models for mobile communication," IEEE Antennas and Propagation Magazine, vol. 45, no. 3, pp. 51-82, 2003.

[19] H. Hashemi, M. McGuire, T. Vlasschaert, and D. Tholl, "Measurements and modeling of temporal variations of the indoor radio propagation channel," IEEE Transactions on Vehicular Technology, vol. 43, no. 3, part 1-2, pp. 733-737, 1994.

[20] F. Babich and G. Lombardi, "Statistical analysis and characterization of the indoor propagation channel," IEEE Transactions on Communications, vol. 48, no. 3, pp. 455-464, 2000.

[21] M. H. Ismail and M. M. Matalgah, "On the use of padé approximation for performance evaluation of maximal ratio combining diversity over weibull fading channels," EURASIP Journal on Wireless Communications and Networking, vol. 2006, Article ID 58501, 7 pages, 2006.

[22] C. Walck, "Handbook on statistical distributions for experimentalists," Internal Report SUF-PFY/96-01, University of Stockholm, Stockholm, Sweden, November 2000.

[23] K. Krishnamoorthy, Handbook of Statistical Distributions with Applications, Chapman \& Hall/CRC, Boca Raton, Fla, USA, 2006.

[24] S. Perras and L. Bouchard, "Fading characteristics of RF signals due to foliage in frequency bands from 2 to $60 \mathrm{GHz}$," 
in Proceedings of the 5th International Symposium on Wireless Personal Multimedia Communications (WPMC '02), vol. 1, pp. 267-271, Honolulu, Hawaii, USA, October 2002.

[25] S. Kourtis and R. Tafazolli, "Evaluation of handover related statistics and the applicability of mobility modelling in their prediction," in Proceedings of the 11th IEEE International Symposium on Personal, Indoor and Mobile Radio Communications (PIMRC'00), vol. 1, pp. 665-670, London, UK, September 2000.

[26] A. H. Zahran, B. Liang, and A. Saleh, "Signal threshold adaptation for vertical handoff in heterogeneous wireless networks," Mobile Networks and Applications, vol. 11, no. 4, pp. 625-640, 2006.

[27] J. Hu, S. Choudhury, and J. D. Gibson, "PSNR. r,f: assessment of delivered AVC/H.264 video quality over 802.11a WLANs with multipath fading," in Proceedings of the 1st Multimedia Communications Workshop (MULTICOMM '06), pp. 1-6, Istambul, Turkey, June 2006.

[28] S. Winkler, Digital Video Quality Vision Models and Metrics, John Wiley \& Sons, New York, NY, USA, 2005.

[29] J. D. McCarthy, M. A. Sasse, and D. Miras, "Sharp or smooth? Comparing the effects of quantization vs. frame rate for streamed video," in Proceedings of the Conference on Human Factors in Computing Systems (CHI '04), pp. 535-542, Vienna, Austria, April 2004.

[30] T. Wiegand, G. J. Sullivan, G. Bjontegaard, and A. Luthra, "Overview of the H.264/AVC video coding standard," IEEE Transactions on Circuits and Systems for Video Technology, vol. 13, no. 7, pp. 560-576, 2003. 